\title{
Propuestas de cambio y debilidad institucional en Chile: De la revuelta social (2019) al inicio del funcionamiento de la Convención Constitucional (2021)
}

\author{
Proposals of Change and Institutional Weakness in Chile: \\ From the Social Revolt (2019) to the Beginning of the \\ Functioning of the Constitutional Convention (2021)
}

\author{
Octavio Avendaño ${ }^{1}$ Nelson Alejandro Osorio Rauld ${ }^{2}$
}

\begin{abstract}
Cómo citar este artículo: Avedaño, O., Osorio Rauld, N. A. (2021). Propuestas de cambio y debilidad institucional en Chile: De la revuelta social (2019) al inicio del funcionamiento de la Convención Constitucional (2021). Revista de Ciencias Sociales Ambos Mundos, (2), 7-18. https://doi.org/10.14198/ambos.20978
\end{abstract}

\begin{abstract}
Resumen
El artículo analiza la precariedad institucional y la escasa capacidad del sistema político chileno para procesar de manera orgánica el malestar que dio origen a la revuelta social que se inicia el 18 de octubre de 2019. Se examinan los factores estructurales, arraigados tanto en el plano económico-social como en el ámbito político-institucional que condujeron al estallido social, además de hechos de tipo coyuntural, derivados de decisiones adoptadas por el actual gobierno de Sebastián Piñera (2018-2022) que tendieron a exacerbar el malestar manifestado en diversos sectores de la población. Se sostiene que ni la revuelta ni las soluciones políticas alcanzadas con posterioridad han logrado revertir la crisis de legitimidad que se había arraigado previamente. Se concluye que este problema podría afectar la estabilidad de un nuevo gobierno, a ser constituido en las próximas elecciones de noviembre, así como al funcionamiento de la Convención Constitucional, comprometiendo la legitimidad de la nueva Constitución.
\end{abstract}

Palabras claves: Chile; revuelta social; crisis de legitimidad; Convención Constitucional; nueva Constitución.

Abstract

This article analyses the institutional precariousness and the weak capacity of the Chilean political system to process through institutional channels the social unrest that triggered the social upheaval that started on October 18th of 2019. Structural factors rooted both in the socio-economic and in the political-institutional fields that led to the social outburst are examined. The conjectural facts, resulted from the decisions adopted by the current government of Sebastián Piñera (2018-2022) that tended to exacerbate the social unrest expressed by diverse sectors of the poulation are also reviewed. It is argued that neither the social upheaval nor the political solutions reached at a later date have achieved to reverse the legitimacy crisis previously rooted. In conclusion, this problem could affect the stability of a new government, to be established after the next elections in November, as well as the functioning of the Constitutional Convention, jeopardizing the new Constitution legitimacy.

Keywords: Chile; social revolt; legitimacy crisis; Constitutional Convention; new Constitution.

1. Octavio Avendaño, Universidad de Chile, Chile. oavendanop@u.uchile.cl https://orcid.org/0000-0001-6945-5327

2. Nelson Alejandro Osorio Rauld, Universidad de Santiago, Chile nelson.osorio.r@usach.cl https://orcid.org/0000-0003-0409-0376

Fecha recepción: 21/09/2021, Fecha aceptación: 18/10/2021

Licencia: Este trabajo está sujeto a una licencia de Reconocimiento 4.0 Internacional de Creative Commons (CC BY 4.0).

https://creativecommons.org/licenses/by/4.0/ 


\section{INTRODUCCIÓN}

Sin duda, una nueva etapa se abre con la revuelta social, iniciada en los primeros días de octubre de 2019 y agudizada desde el día 18 de ese mismo mes. Las protestas y movilizaciones sociales que se expandieron por todo Chile, y que solo lograron ser aplacadas recién a partir de la primera quincena de marzo de 2020, por las condiciones de autocuidado y de control interno que impuso la pandemia, remecieron las bases institucionales del sistema político. A su vez, esas mismas protestas y movilizaciones interpelaron directamente la lógica de funcionamiento del modelo de desarrollo, que si bien fue impuesto bajo condiciones autoritarias, fue mantenido en lo esencial por los gobiernos que se sucedieron desde 1990 hasta la fecha.

La revuelta social logró involucrar una diversidad de actores, movilizados por distintas causas, y por el carácter transversal de la protesta. Rasgo que se mantuvo, al menos, hasta las primeras semanas del 2020, debido a que a partir de ese entonces las movilizaciones fueron adquiriendo una mayor radicalidad, siendo la tónica el enfrentamiento con la fuerza pública. Sin embargo, desde el mismo 18 de octubre se puso en evidencia la falta de conducción, el predominio de manifestaciones espontáneas, el uso reiterado de la violencia en diferentes puntos de Santiago y del territorio nacional, así como el cuestionamiento a todo intento de articulación y representación, ya sea de los partidos y de organizaciones ciudadanas -como las reunidas en la Mesa de Unidad Social- que de manera infructuosa intentaron darle direccionalidad y orientación al movimiento.

Algunas de las interpretaciones que emergieron al calor de los acontecimientos, apuntaron a destacar el tema de las pulsiones, debido al supuesto perfil juvenil de los manifestantes, y el carácter 'anómico' de gran parte de las manifestaciones (Peña, 2020). También se ha hecho mención a que la revuelta ocurre a raíz de una falta de eficiencia entre los tomadores de decisiones, haciendo analogía entre lo ocurrido y lo que significó la explosión del reactor nuclear de Chernóbil, en 1986 (Tironi, 2020, pp. 20-23). Otras interpretaciones, en cambio, se empeñaron en resaltar la configuración de un "nuevo pueblo" (Ruiz, 2020) que se había ubicado en una posición antagónica hacia el conjunto de las élites.

Pero desde nuestra interpretación lo que realmente aflora, a propósito de la revuelta, y en los posteriores eventos definidos para canalizar políticamente la crisis, es la precariedad institucional y la escasa capacidad para procesar de manera más orgánica aquel "malestar" existente antes y des- pués de octubre de 2019. En otras palabras, ni la revuelta ni los acontecimientos que políticamente acompañan la llamada "solución constitucional" logran revertir la crisis de legitimidad existente previamente. Por el contrario, y como queda claramente reflejado en la elección de constituyentes, gobernadores y autoridades municipales durante los días 15 y 16 de mayo del presente 2021, la falta de legitimidad persiste y se sigue proyectando, como ha ocurrido también en otros escenarios latinoamericanos (Brink, et al., 2019). Incluso, es posible sostener que ese mismo problema se podría proyectar tras el inicio del funcionamiento de la Convención Constitucional y la elección de un nuevo gobierno, a ser constituido luego de las próximas elecciones de noviembre del año en curso.

\section{LAS RAZONES DEL "OCTUBRE CHILENO"}

El estallido social es, en gran medida, el resultado de una serie de tensiones no resueltas (PNUD, 2019). Como se ha indicado en trabajos anteriores, algunas de estas tensiones obedecieron a factores de tipo estructural, o a fenómenos negativos que se fueron arraigando por décadas, mientras que otras fueron coyunturales, derivadas del estilo de gestión que impuso el segundo gobierno de Sebastián Piñera (2018-2022) (Avendaño, 2019; Avendaño y Escudero, 2020). A su vez, dentro de los factores estructurales se distinguen los que son económico-sociales de aquellos de carácter político e institucional (Avendaño, 2019; Osorio 2019a).

Partiendo por los factores estructurales, el primero de ellos son los efectos desarticuladores que trajo consigo la modernización neoliberal, como el aumento de la precariedad laboral, el predominio de la inseguridad social, la nueva pobreza y la agudización de la desigualdad. De un 40\% de pobreza existente en 1990 , ella se reduce a un $8,6 \%$ hacia 2018. Pese a esta importante disminución hacia esa misma fecha, en 2016, el coeficiente Gini llegaba a 47,7 , lo que situaba a Chile como uno de los países más desiguales de la región (OCDE, 2018). La mala distribución de los recursos también se puede observar en la concentración de la riqueza, en la que poco antes del estallido social, el 1\% más rico había logrado capturar cerca del 33\% de lo producido por la economía nacional; al mismo tiempo, el 0,1\% de la población concentraba el 19,5\% del ingreso (PNUD, 2017a; Ministerio de Desarrollo Social, 2018).

Por otra parte, el aumento de la desigualdad ha traído consigo otro tipo de situaciones expresadas 
en el plano material y a nivel de la subjetividad. Basta señalar la segmentación que se ha venido generando en el sistema educacional y el problema del endeudamiento en los estudiantes universitarios (Bellei, 2016; Donoso, 2017). En efecto, la solución bancaria al problema del acceso a la universidad provocó un conflicto mayor asociado a la frustración en las expectativas y economías familiares de los estudiantes, que llegaban a destinar buena parte de los salarios para pagar matrículas y deudas (Fundación Sol, 2016). Siempre en el plano de lo material, se podría mencionar lo que ha llegado a ser el agobio en las ciudades del país, debido a los efectos de la segregación urbana, la creciente especulación del valor del suelo, las dificultades de acceso a la vivienda de sectores de más bajos ingresos y los problemas de seguridad ciudadana (Rasse, 2016; Vergara, 2019). En el plano más subjetivo, el contraste entre la sanción para el resto de la población y el tipo de penas recibidas por los responsables de la colusión, situaciones de corrupción y de otro tipo de delitos cometidos por miembros de la élite, fue generando y acumulando una sensación de injusticia e impunidad (Güell, 2019).

Dentro de los factores estructurales es posible reconocer, en segundo lugar, el diagnóstico de un malestar que se advierte en el período inmediato a la recuperación democrática. En 1998, el Informe de Desarrollo Humano del Programa de Naciones Unidas para el Desarrollo (PNUD, 1998) pudo constatar un malestar arraigado en importantes segmentos de la población, no obstante el aumento de las oportunidades y la mejora experimentada en sus condiciones de vida. Esta "paradoja" que traía consigo el proceso de modernización no pasó desapercibida en la discusión pública, al coincidir en el mismo año con un intenso debate suscitado en la coalición de gobierno, la Concertación de Partidos por la Democracia, pese a que en lo inmediato el diagnóstico no se tradujo en un cambio sustantivo en la orientación de las políticas públicas (Avendaño, 2017; Huneeus, 2014).

El creciente malestar, unido al aumento de la desigualdad, configuraron un tercer factor que decantó en movimientos de protestas que fueron adquiriendo un carácter cada vez más masivo y transversal. Tal fue lo ocurrido con la irrupción del movimiento de estudiantes secundarios, durante el primer semestre de 2006, cuyo principal propósito fue la derogación de la Ley Orgánica Constitucional de Educación (LOCE), cuya puesta en vigencia se había producido un día antes de la finalización del régimen autoritario, el 10 de marzo de 1990. Además, el movimiento de estudiantes secundarios, expresó una fuerte crítica hacia el lucro y la mercantilización de la educación (Donoso, 2017, p. 76; Mayol, 2012).

Durante los primeros meses de 2007, se desencadenaron protestas espontáneas en diferentes puntos de la Región Metropolitana por parte de los usuarios del trasporte público y la locomoción colectiva, que fueron reflejo de la insatisfacción de los usuarios frente al inicio de la puesta en marcha del nuevo sistema de trasporte, más conocido como Transantiago (Mardones, 2007). En los primeros meses, la fallida puesta en marcha de este nuevo sistema de trasportes afectó el traslado de los habitantes de las comunas periféricas y de más bajos ingresos, situación que se prolongó por casi dos años, pese al enorme caudal de recursos públicos introducidos para intentar mejorarlo (Ureta, 2017).

Por intensidad, duración e impacto político, las mayores movilizaciones se registraron durante todo el año 2011 (Segovia y Gamboa, 2012; Varas, 2013; Avendaño, 2013, 2014). Estas se iniciaron, en los primeros días de febrero, con la reacción de los habitantes de Magallanes ante la decisión de la autoridad de aumentar el precio del gas, un recurso vital para los habitantes de esa región (Cofré, 2016; Romero, 2015). Las protestas ciudadanas fueron de tal magnitud que en el lapso de un mes obligaron al gobierno a revocar dicha medida y a cambiar al ministro de Energía de aquel entonces. De manera casi simultánea, en el mes de marzo se desencadenaron movilizaciones y movimientos de protesta en Calama debido a la escasa inversión de recursos públicos, de una de las zonas que más genera divisas para el gobierno central. La irrupción de estos movimientos regionales se hizo extensivo hasta los meses de marzo y abril, a raíz del estallido de protestas en Aysén. Protestas similares ocurrieron en la localidad de Freirinas, en la región de Atacama, a propósito de la contaminación de la planta faenadora de cerdos perteneciente a la empresa Agrosuper. Al igual que en los casos anteriores, el estallido de Aysén se caracterizó por convocar al conjunto de los habitantes de esa zona, por la emergencia de líderes locales y la inexistente mediación de los partidos (Cofré, 2016). Lo ocurrido en Aysén y Freirina por aquellos días estuvo marcado por un fuerte despliegue policial, cuyos efectivos se enfrentaron de manera directa a la población movilizada, generando con ello una gran conmoción nacional.

De todas las movilizaciones efectuadas en 2011, las relacionadas con temas ambientales y el problema educacional resultaron ser las más masivas y replicadas en las principales ciudades -capi- 
tales provinciales y regionales- a lo largo del país (Segovia y Gamboa, 2012; Avendaño, 2014). Las marchas efectuadas semanalmente por el Movimiento Patagonia sin Represas, concitaron un importante apoyo, inédito hasta ese entonces por causas ambientales. En años anteriores, las protestas y conflictos ambientales habían tenido un carácter estrictamente local, debido a la instalación de vertederos o industrias contaminantes, cuyo efecto era percibido solo por los habitantes de poblaciones y asentamientos colindantes. Patagonia sin Represa logró visibilizar los problemas ambientales, asociados a grandes proyectos de inversión hidroeléctrica, generando con ello una mayor conciencia por la defensa del patrimonio ambiental en distintos territorios del país (Silva, 2016).

En el caso de las movilizaciones por la educación, estas también comenzaron en el mes de abril de 2011 y se extendieron hasta los inicios de 2012, involucrando tanto a estudiantes universitarios, secundarios, docentes y a una diversidad de organizaciones culturales y barriales (Donoso, 2017, p. 83). Para el gobierno de turno, el primero de Sebastián Piñera (2010-2014), el costo político fue enorme, debiendo introducir cambios al gabinete y el reemplazo de tres ministros de educación dentro del mismo año (Varas, 2013; Avendaño, 2014). En el mes de agosto de 2011, el presidente Piñera registró el nivel más alto de desaprobación que un gobierno y un mandatario registraran desde el retorno de la democracia.

Aunque con menor intensidad, las movilizaciones por la educación se extendieron hasta fines del mandato del presidente Piñera, cuyas demandas fueron acogidas en las propuestas del programa de gobierno de la Nueva Mayoría - coalición de centroizquierda que agrupaba a los partidos de la ex Concertación junto al Partido Comunista (PC) -, presidido, por segunda ocasión, por la presidenta Michelle Bachelet (2014-2018). Las movilizaciones de 2011 lograron sobrepasar las formas de protestas y conflictos sociales registrados con anterioridad. En primer lugar, porque introdujeron demandas más amplias e incluso con contenidos universales, superando con ello el carácter sectorial de los conflictos anteriores. En segundo lugar, porque esas demandas incluían aspectos relacionados con una mayor inclusión, la superación de las diferencias y la segmentación, así como la crítica a la mercantilización de la educación en todos sus niveles (Bellei, 2016, p. 234). Fueron promovidas propuestas relacionadas con gratuidad universal y fin al lucro en la Educación. En tercer lugar, formularon una fuerte crítica e interpelación al modelo de desarrollo y al funcionamiento del sis- tema político. Se puso en evidencia la crisis de representación que se venía arrastrando, de manera paulatina, desde la década anterior. Fue así que, con el fin de revertir los problemas del funcionamiento del sistema político, surgieron propuestas en torno a la realización de una asamblea constituyente y una nueva carta fundamental (Escudero, 2021).

El segundo gobierno de Michelle Bachelet intentó responder a muchas de las demandas que surgieron a partir de las movilizaciones de 2011 y que se proyectaron hasta fines de 2013 (Moreno, 2018, p. 49). Sin embargo, tensiones que surgieron al interior de la coalición de gobierno, la Nueva Mayoría, generaron lentitud en la implementación de ciertas reformas, en el ámbito educacional, y pusieron en alerta al conjunto de la oposición de los partidos de derecha (Avendaño y Escudero, 2019). Estos se lograron vincular con agrupaciones de padres y apoderados para evitar un proyecto que ponía fin al copago y a la selección escolar ${ }^{1}$. A su vez, la gratuidad universitaria, que se suponía sería universal, había logrado beneficiar solo a un 60\% de los estudiantes más vulnerables del país, es decir, un segmento del universo total de estudiantes (Arzarola, 2019, p. 6). El gobierno contemplaba además promover un proceso constituyente con miras a lograr un cambio de la Constitución (Viera, 2020, pp. 53-54).

Las mismas tensiones aludidas en el párrafo anterior, que surgieron al interior de la coalición de gobierno, además de la férrea oposición de partidos de Chile Vamos y también del gran empresariado (Osorio, 2019c), dificultaron el proceso de cambio constitucional. Durante el 2016, el gobierno logró llevar a cabo la realización de cabildos autoconvocados, a nivel local, provincial y regional, logrando una participación de $\mathbf{2 0 0}$ mil personas a nivel nacional (Escudero et al, 2020). Con la información sistematizada se logró la elaboración de un proyecto considerado limitado por muchos de quienes respaldaron inicialmente la iniciativa de los cabildos, sin que fuera puesto en la discusión legislativa, en el segundo mandato del gobierno del presidente Sebastián Piñera (2018-2022).

Por otra parte, durante el segundo año del gobierno de Bachelet se dieron a conocer varios casos de corrupción, que afectaron inicialmente a repre-

1. Para ver la vinculación entre derecha y agrupaciones de padres y apoderados, véase el siguiente enlace: https:// www.latercera.com/noticia/udi-en-marcha-con-apoderados-de-subvencionados-queremos-reforma-pero-no-de-esta-forma/ visitado el día 10 de mayo de 2021. 
sentantes de los partidos de derecha, debido a irregularidades detectadas en el financiamiento de las campañas. De manera simultánea, se produjo un hecho ligado al uso de información privilegiada, que afectó al entorno de la familia de la presidenta Bachelet. Estos hechos, más el aumento de casos vinculados al financiamiento ilícito, dieron cuenta de la estrecha relación que existía entre el mundo de los negocios, de los grandes empresarios, con las campañas electorales (Huneeus y Avendaño, 2018, pp. 180-181; Osorio, 2019b).

El cuarto factor fue la crisis institucional que se fue registrando a partir del año 2007. Hasta ese entonces, se había configurado una "institucionalidad con pies de barros" (Altman y Luna, 2011), arrastrada de las situaciones deficitarias en el régimen democrático chileno, que hizo que la crisis se hiciera irreversible tras la ola de movilizaciones y protestas del 2011. Una de las manifestaciones de la crisis fue la desafección ciudadana hacia el sistema político, la baja confianza interpersonal y hacia las instituciones democráticas (Huneeus, 2014; Venegas, 2016). También ha sido expresión de la crisis el descenso de la participación electoral, en comparación con aquella registrada en los comicios que siguieron inmediato al plebiscito de 1988 (Morales, 2018). Esta ha caído sistemáticamente de un $87 \%$ de participación en la elección presidencial y legislativa de 1989 , a menos del $50 \%$ de en 2012, tras la implementación de la reforma electoral de inscripción automática y voto voluntario (Ruiz y Osorio, 2016). Desde esa fecha hasta 2019, la participación ha seguido disminuyendo, incluso llegando a cifras alarmantes, como fue la elección de alcaldes y concejales en 2016, al llegar al 36\% (PNUD, 2017b, p. 196). Cabe mencionar que esta cifra sólo aumentó a poco más de un 50\% de participación para la segunda vuelta de la contienda presidencial de 2017 y para el plebiscito nacional para la nueva Constitución de 2020; sin embargo, la participación electoral volvió a registrar una caída para la elección de constituyentes en 2021.

Otra importante manifestación de la crisis institucional se ha manifestado, sobre todo, en el ámbito de la representación política. La baja valoración que en los años noventa registraron el Congreso y los partidos fue dando paso a una fuerte caída en la identificación entre la ciudadanía y este tipo de colectividades. Tal fenómeno afectó por igual a los partidos de la totalidad del espectro, desde la izquierda a la derecha. A ello se agrega el debilitamiento organizativo que también afecta al conjunto de los partidos actualmente existentes. Situación que se expresa en la pérdida del carácter nacional (desnacionalización) de la totalidad de los partidos, muchos de los cuales concentran su representación en determinadas regiones y territorios, la existencia de estructuras precarias y una fuerte dependencia con los parlamentarios (Gamboa, et al., 2016, p. 8).

Las reformas políticas introducidas entre 2014 y 2016, que modificaron la ley sobre partidos, regularon el financiamiento y reemplazaron el sistema electoral binominal por un sistema proporcional (Ruiz y Osorio, 2016), no lograron revertir dicha crisis sino que, por el contrario, en el caso de esta última, se tendió agudizar el debilitamiento de los partidos (Avendaño, 2017). Una clara demostración fue el aumento de la fragmentación producida por los resultados de la contienda electoral de 2017. Como lo demuestra Miranda y López (2020), en 2017, 26 partidos se presentaron a la elección de la Cámara Baja, de los cuales 16 obtuvieron cargos de diputados. Esta situación contrasta con lo registrado entre 1989 y 2013, con un promedio de 10 partidos en competencia y 8 con cargos electos. Si bien es cierto, el cambio de sistema electoral facilitó la emergencia de una tercera fuerza, ésta no logró superar la pérdida de legitimidad y valoración negativa que la población ha venido manifestando frente a los partidos.

A los factores de tipo estructural, se añaden también aquellos de tipo coyuntural. Vale decir, factores derivados del estilo de gestión y de las decisiones adoptadas por el actual gobierno de Sebastián Piñera (Avendaño, 2019). Desde un comienzo, el gobierno promovió un estilo de gestión tecnocrática que combinó con la adopción de métodos autoritarios, a fin de aplacar movilizaciones y protestas que pudieran generar efectos desestabilizadores. Sin ir más lejos, uno de los hechos que provoca las protestas de estudiantes y usuarios del Metro fue la decisión de subir el billete de este medio de la locomoción colectiva, aludiendo a razones estrictamente técnicas y a la decisión adoptada por un grupo de expertos ${ }^{2}$.

En cuanto a las medidas autoritarias, desde el Ministerio de Interior se desplegaron una serie de operativos en la zona de la Araucanía, que derivaron en enfrentamientos y en hechos trágicos como la muerte de Camilo Catrillanca, en la comunidad de

2. Para ver la solución tecnocrática del gobierno del presidente Piñera al asunto de la tarifa del metro, véase el siguiente enlace: https://www.emol.com/noticias/ Nacional/2019/10/05/963355/Metro-Transantiago-tarifa-transporte-publico.html visitado el día 12 de junio de 2021. 
Temucuicui $^{3}$. Durante el año 2019, se intensificaron las protestas de profesores y estudiantes. Los profesores llevaron a cabo jornadas de paralización durante todo el primer semestre de ese año, sin lograr respuesta a las demandas formuladas en su petitorio. Además, marchas y manifestaciones callejeras, convocadas por el Colegio de Profesores, fueron reprimidas por las fuerzas policiales. Los estudiantes de establecimientos municipalizados y liceos emblemáticos, que demandaban mayores recursos y mejoras en la infraestructura, fueron igualmente reprimidos y sometidos a una política de control a través de un proyecto de "Aula Segura", que facultaba a los directivos a expulsar a estudiantes involucrados en hechos de violencia. Sin embargo, por sobre mejorar la convivencia escolar, el proyecto buscaba aplacar movilizaciones que durante casi dos años llevaron a cabo estudiantes de los establecimientos mencionados, tanto en Santiago como en otras comunas de la Región Metropolitana.

Bajo esas condiciones, no fue casual, entonces, que hayan sido los estudiantes quienes asumieron el mayor protagonismo en las protestas tras el anuncio del alza del pasaje del Metro. Y, como en otras experiencias históricas, como la "revolución de la chaucha" de 1949, o los sucesos del 2 y 3 de abril de 1957 (Moulian, 2006, 2020), no fue coincidencia tampoco que un alza en los precios de la locomoción colectiva derivara en protestas ciudadanas y en una cuasi insurrección popular (Salazar, 1990, p. 260ss.).

Hacia el día 18 de octubre, la protesta ciudadana derivó en una auténtica revuelta popular, que implicó la suspensión de la locomoción colectiva, cortes de calle y protestas espontáneas que se intensificaron al caer la noche (Fernández, 2020, p. 26). También se intensificaron las acciones de violencia. Esa misma noche manifestantes supuestamente incendiaron el edificio corporativo de la empresa ENEL y 20 estaciones del Metro, mientras 41 sufrieron diversos daños ${ }^{4}$. Frente a este tipo de acciones, el gobierno decretó Estado de Excepción Constitucional,

3. Véase en el siguiente enlace de CIPER los vídeos de la muerte de Camilo Catrillanca https://www.ciperchile.cl/2018/12/19/muerte-de-catrillanca-ciper-revela-en-exclusiva-tres-de-los-videos-que-grabo-carabineros/ visitado el día 3 de julio de 2021.

4. Sobre la violencia del estallido, véase el siguiente enlace https://www.biobiochile.cl/noticias/nacional/regionmetropolitana/2019/10/19/20-estaciones-quemadasy-41-con-diversos-danos-el-recuento-de-metro-porjornadas-de-protestas.shtml visitado el día 3 de abril de 2021 dejando a la Región Metropolitana bajo control de los militares ${ }^{5}$. Pero lejos de frenar la revuelta social, la decisión tomada por el gobierno trajo un inmediato efecto explosivo al extender las protestas y las movilizaciones a lo largo de todo el país.

\section{DE LA SALIDA POLÍTICA DE LA REVUELTA AL "PLEBISCITO DE ENTRADA"}

Las manifestaciones callejeras, concentraciones y protestas, continuaron hasta la primera quincena de marzo de 2020, viéndose interrumpida con el fenómeno de la pandemia y la decisión del gobierno de establecer cuarentena en diversas ciudades del país, para lo cual decretó nuevamente el Estado de Excepción. Entre el 19 de octubre y los primeros días de marzo de 2020, diversas fueron las manifestaciones y, a su vez, variable fue su intensidad. En ciudades como Antofagasta, Valparaíso, Talca, Concepción, Valdivia y Santiago, los hechos de violencia continuaron de manera focalizada, centrándose en determinados territorios y en un perfil de manifestantes marcadamente juvenil. Durante las primeras semanas que siguieron al 19 de octubre, las protestas tuvieron un carácter masivo y, desde el punto de vista social, transversal en su convocatoria.

El viernes 25 de octubre tuvo lugar la marcha más multitudinaria de la que se tenga registro, en la cual alrededor de un millón doscientas mil personas se congregaron en todas las arterias que convergen al sector de Plaza Italia, en la ciudad de Santiago, bautizada en esos días con el nombre de "Plaza de la Dignidad" (Avendaño, 2019, p. 115; Fernández, 2020 , p. 38). Pero las manifestaciones y protestas ciudadanas tendieron a descender hacia la segunda mitad del mes de noviembre siendo en un comienzo revitalizadas por la irrupción de un movimiento feminista, en torno al colectivo Las Tesis, que se replicó en todo el país y con gran impacto en el resto del mundo. En los meses de enero y febrero, contra todo pronóstico, las protestas continuaron logrando incluso aparecer en todo tipo de eventos públicos, como conciertos, eventos deportivos, festivales,

5. Véase la ley de Declaración de Estado de Excepción en toda la Región Metropolitana en el siguiente enlace https://www.interior.gob.cl/transparenciaactiva/doc/ ActosyDocumentosDiarioOficial/200/6849284.pdf visitado el día 22 de abril de 2021 
centros comerciales, o donde simplemente hubiese algún tipo de aglomeración.

En los momentos de mayor masividad y protesta ciudadana, que se extendió por casi un mes, las movilizaciones destacaron por su espontaneidad y por carecer de toda conducción. Al igual como ocurrió durante el 2011 la clase política se vio sobrepasada y se puso en evidencia la debilidad de los partidos de oposición (Avendaño y Escudero, 2019; Escudero y Olivares, 2021). El gobierno, en los primeros días, anunció medidas como la suspensión del alza de los pasajes, aumento de la pensión básica solidaria, medidas que no lograron receptividad en la población. Por el contrario, las protestas continuaron e intensificaron. También se realizaron encuentros entre el gobierno y algunos representantes de la oposición, vinculados a la Democracia Cristiana y al Partido por la Democracia, sin que eso fuera asumido por el resto de los partidos de centroizquierda e izquierda.

En paralelo, fueron promovidas otras acciones de parte de la Mesa de Unidad Social, orientadas a canalizar parte de las demandas y del descontento, considerando que ella se había constituido a partir de la iniciativa del Movimiento No+AFPs, el Colegio de Profesores, la Central Unitaria de Trabajadores, y otras agrupaciones locales, en los días en que estaba en pleno desarrollo del conflicto entre el Magisterio y el Ministerio de Educación. Pese a estar alejada de la influencia de los partidos de centroizquierda más tradicionales, la Mesa de Unidad Social tampoco logró darle conducción a la revuelta.

En la jornada del 12 de noviembre se registraron los hechos más violentos desde el comienzo de la revuelta. A tal nivel de intensidad llegaron los hechos, que diferentes medios de prensa, nacional y extranjera, hicieron mención a los efectos desestabilizadores que estaba generando la revuelta. Cifras extraoficiales daban a conocer un saldo de entre 30 a 40 muertos, desde la noche del 19 de octubre. Por cadena nacional de televisión, el presidente Piñera se refirió a la decisión del gobierno de reintegrar a militares y carabineros en retiro, con el fin de frenar la ola de disturbios y protestas que se estaba viviendo en todo el país ${ }^{6}$. También hizo un llamado a un acuerdo, entre gobierno y oposición, con miras a lograr la paz y proponer un cambio constitucional.

6. Para ver el llamado del Presidente Piñera a lograr acuerdos para salir de la crisis véase el siguiente enlace https://www. emol.com/noticias/Nacinal/2019/11/12/967033/Presidente-Pinera-acuerdos-manifestaciones-Constitucion. html visitado el día 2 de mayo de 2021.
Desde el oficialismo hasta los sectores de la oposición de centroizquierda acogieron el llamado y la noche del 15 de noviembre suscribieron el 'Acuerdo por la Paz y la Nueva Constitución'. Formaron parte y suscribieron ese acuerdo, los partidos Renovación Nacional (RN), Unión Demócrata Independiente (UDI) y Evolución Política (EVOPOLI), por parte del oficialismo, además de la Democracia Cristiana (DC), el Partido por la Democracia (PPD), el Partido Socialista (PS), Comunes, Revolución Democrática (RD) y el Partido Liberal (PL). Gabriel Boric, uno de los principales dirigentes de Convergencia Social (CS), perteneciente al Frente Amplio (FA), firmó a título personal, en calidad de independiente. Se restaron del acuerdo el Partido Comunista (PC), el Partido Humanista $(\mathrm{PH})$ y la Federación Regionalista Verde Social (FRVS) (Escudero y Gajardo, 2020, p. 4; Heiss, 2020, pp. 106-107). Como parte del acuerdo se estableció que se partiría de una hoja en blanco y que las decisiones se tomarían con un quorum de 2/3.

Con el acuerdo se dio inicio a una nueva fase, en la que una parte de la oposición desestimó el camino de la protesta con miras a establecer las condiciones para asumir el cambio de la nueva Constitución. Esto significó la creación de una comisión técnica, en la que participaron representantes de todos los partidos que suscribieron el acuerdo del 15 de noviembre. La revuelta social se había iniciado con la formulación de una serie de demandas económico-sociales, que pusieron en cuestión el funcionamiento del modelo de desarrollo. Además, como ya se ha dicho, no hubo posibilidad de conducir la revuelta antes del acuerdo del 15 de noviembre. Con el acuerdo, se ofrecía una salida política a la crisis mediante el cambio de la Constitución, sin asumir de manera directa un compromiso frente a las demandas que quedaban postergadas.

La mencionada comisión estableció una serie de condiciones para definir los procedimientos y la modalidad del cambio de la Constitución. Descartada la posibilidad de una Asamblea Constituyente el mismo día del acuerdo, la comisión estableció la posibilidad de dos mecanismos de representación para la elaboración de la nueva Carta Fundamental: a través de una Convención Mixta, integrada por parlamentarios y representantes electos, o bien, mediante una Convención Constitucional, que estaría compuesta, en su totalidad, por miembros electos. También propuso la realización de un plebiscito de entrada -en el que la ciudadanía, mediante dos papeletas, debería optaría en la primera por el apruebo o el rechazo a una nueva Constitución y en la segunda decidir por Convención Mixta o Conven- 
ción Constitucional- y otro de salida a realizar al momento de haberse redactado el documento de la nueva Carta Fundamental. En el marco de las negociaciones que se dieron en la comisión técnica, surgió la posibilidad de otorgar cupos especiales a los pueblos originarios y darle un carácter paritario al órgano de representación encargado de discutir y elaborar la nueva Constitución.

Diversos estudios de opinión efectuados entre diciembre de 2019 y marzo de 2020 mostraron un amplio respaldo a la opción apruebo, aunque con un porcentaje mucho más bajo hacia la Convención Constitucional (Barómetro del Trabajo, 2020; Criteria, 2020; Data influye, 2020). Aprobada la reforma constitucional que permitiría dar inicio al proceso constituyente, con la convocatoria a plebiscito, se acordó que éste se llevaría a cabo a fines de abril de 2020. Sin embargo, el inicio de la pandemia, y el establecimiento de la cuarentena en diversas comunas del país, interrumpieron la campaña que tanto los partidos de oposición como las organizaciones vinculadas a Unidad Social dieron inicio en el mes de enero de 2020. El gobierno decidió postergar el plebiscito hasta una nueva fecha, la cual quedó fijada para el 25 de octubre de ese año.

Por razones que se exponen en el siguiente apartado, la oposición realizó una campaña dividida a favor de la opción Apruebo y por la Convención Constitucional. Pese a ello, estaban dadas todas las condiciones para un triunfo favorable a los partidarios del cambio constitucional, en gran medida, porque la pandemia reafirmó gran parte de las demandas con las cuales se produjo la revuelta social. En el plebiscito del 25 de octubre, de los votos válidamente emitidos, el $78,3 \%$ se inclinó a favor de la oposición "Apruebo" y el 21,7\% por "Rechazo". A su vez, el 78,98\% lo hizo a favor de la Convención Constitucional y 21,02\% por la Convención Mixta. Contra todo pronóstico, no hubo voto cruzado de parte de quienes se inclinaron por la opción "Apruebo".

\section{LAS TENSIONES EN LA OPOSICIÓN Y EL DEVENIR DE LA CONVENCIÓN CONSTITUCIONAL}

Con el acuerdo del 15 de noviembre se evidenciaron dos "almas" en la oposición. Una dispuesta a lograr entendimientos con el gobierno, junto con respaldar su agenda legislativa y otras medidas que se adoptarían a partir del inicio de la pandemia. La otra, que se excluyó del acuerdo, estuvo representada por el
PC, una parte del FA, el PH y las organizaciones que conformaban la Mesa de Unidad Social. Estos dos "bloques" derivados del 15 de noviembre se desdibujaron al momento de definir una plataforma unitaria de la oposición. Desde sectores del FA se planteó la necesidad de definir "mínimos comunes" antes de cualquier pacto o alianza electoral ${ }^{7}$.

Estas exigencias fueron asumidas por la totalidad de los dirigentes del FA, incluyendo aquellos que habían pertenecido a la Nueva Mayoría, la coalición de centroizquierda que, como se mencionó anteriormente, estuvo en el gobierno en el período 2014-2018. A medida que se acercaba la inscripción de primarias para las elecciones de gobernadores y alcaldes, la alianza entre esas dos fuerzas políticas fue desechada y luego reactivada. Horas antes de que el plazo expirara, el FA en conjunto con el PC, decidieron descartar dicha alianza inscribiendo por separada su propia lista. Después de una historia de desencuentros, que se remontaba a los inicios del FA, en enero de 2017, éste conglomerado junto con el PC constituían una alianza autocalificada de "antineoliberal".

Tanto los dirigentes de la ex Concertación (DC, PPD, PS y PR), como los del PC y del FA evitaron referirse a los entretelones del frustrado acuerdo, indicando la posibilidad de retomar las negociaciones en vísperas a la elección de los convencionales. Al final, tampoco prosperó dicho acuerdo y tanto la ex Concertación, denominada ahora Unidad Constituyente, como la nueva alianza PC-FA, denominada Apruebo Dignidad, optaron por caminos separados para enfrentar lo que sería una "mega-elección". Esta "mega-elección", denominada así porque los ciudadanos deberían escoger en cuatro papeletas distintos candidatos a convencionales, gobernadores, alcaldes y concejales, tuvo lugar los días 15 y 16 de mayo. En ella no solo participaron listas de las dos alianzas mencionadas, también lo hicieron listas de independientes y de otras agrupaciones de izquierda. La derecha, por su parte, fue unida en las elecciones de convencionales pero separada en las de gobernadores. En la elección de los 155 convencionales, la derecha no fue capaz de alcanzar el tercio de la representación, al ser elegidos 37 convencionales.

Por otra parte, 28 representantes obtuvo la lista Apruebo Dignidad, gracias al buen desempeño de

\footnotetext{
7. Para más información, véase https://www.elmostrador. cl/dia/2020/09/28/frente-amplio-ahora-se-abre-a-primarias-con-el-resto-de-la-oposicion-pero-exige-minimosprogramaticos-y-eticos/ visitado el día 14 de julio de 2021
} 
Revolución Democrática, que obtuvo 9 cupos, mientras el PC logró la no despreciable cifra de 7 convencionales. Unidad Constituyente obtuvo 25 convencionales, siendo el partido más afectado la DC, que sólo logró dos cupos en la Convención, uno de los cuales se presentaba en calidad de independiente. La gran sorpresa estuvo de parte de la Lista del Pueblo que logró 11 convencionales. En la "mega-elección" se consolidó la alianza PC-FA debido al resultado obtenido en la elección de convencionales. La Unidad Constituyente, que tuvo un magro resultado en la elección de convencionales, tuvo un mejor desempeño en las de gobernadores y municipales.

Algunos exponentes del "neoinstitucionalismo", entre los que sobresalen Mahoney y Thelen (2010), han demostrado que, por lo general, los procesos de cambio adoptan ritmos graduales, superponiendo y aprovechando los logros de reformas anteriores. Así ocurrió con la reforma que hizo posible pasar del sistema electoral binominal a uno proporcional, permitiendo la emergencia de una tercera fuerza, el FA en las elecciones de 2017 (Avendaño y Escudero, 2019). Así también ocurrió con el acuerdo del 15 de noviembre y la labor emprendida por la comisión técnica, que permitieron la elección de una Convención Constitucional paritaria y que incluye a 17 representantes de los pueblos originarios.

Las mismas decisiones adoptadas por la Comisión Técnica, respecto a la confección de las listas, junto a la existencia del proporcional, hicieron posible que en la Convención estén representados los independientes y quienes no participaron, como la Lista del Pueblo y el PC. Sin embargo, desde las elecciones de 2017 , hasta la segunda vuelta de gobernadores efectuada el día 13 de junio, es posible constatar una situación de volatilidad que redunda también en inestabilidad. A su vez, las fuerzas políticas emergentes que se logran posicionar electoralmente, desafiando y desplazando a los partidos tradicionales, como ocurrió inicialmente con el FA y luego, en la elección de convencionales, la Lista del Pueblo, no logran revertir ni la crisis institucional ni el problema de legitimidad que afecta al sistema político. La falta de solidez institucional se puede convertir en un factor decisivo para el éxito del proceso constituyente $y$, sobre todo, para el arraigo y la legitimidad de la nueva Constitución.

\section{REFLEXIONES FINALES}

De acuerdo a lo expuesto, podemos afirmar que la revuelta social que tuvo lugar en Chile a partir del
18 de octubre de 2019, representa uno de los puntos de inflexión más importantes desde el inicio de la transición a la democracia. En ella se manifiesta una aguda crítica al funcionamiento del sistema político y, al mismo tiempo, al modelo de desarrollo socioeconómico impuesto por la dictadura militar y corregido por los gobiernos posautoritarios. El carácter explosivo y violento de la revuelta social se explica, fundamentalmente, por una acumulación de factores "estructurales", como la alta desigualdad, un profundo malestar y una crisis institucional, fenómenos que han aportado en desatar un proceso de movilización social que se viene registrando con más intensidad a partir de 2011, cuyo climax fue el estallido social del 18 de octubre de 2019. Se suman a esos factores, algunos hechos coyunturales, relacionados con el estilo de gobierno del presidente Piñera, administración que ha tomado algunas decisiones con orientación autoritaria y tecnocrática que han profundizando el malestar y la desafección existentes desde hace décadas en la ciudadanía.

Para solucionar la "crisis integral", parte de la élite política decidió optar por una salida institucional que permitió el inicio de un inédito proceso constituyente con miras a elaborar una nueva Constitución. A nuestro juicio, esta "solución constitucional" - con fuerte contenido democrático- permitió canalizar buena parte de las demandas de cambio político que se manifestaron en el estallido y las movilizaciones post 18 de octubre. No obstante, al mismo tiempo, se puede observar que el sistema político arrastra una notoria debilidad institucional, sobre todo por su escasa capacidad, energía y solidez para procesar las demandas económico-sociales urgentes requeridas por los chilenos en la revuelta social. Dicha postergación no ha contribuido en subsanar el problema del malestar existente antes de las movilizaciones. Por el contrario, el problema sigue existiendo pese a que no se expresa en movimientos de protesta, frenados producto de las condiciones de autocuidado y de control interno que ha impuesto la pandemia, sino que más bien se manifiesta en una falta de confianza y legitimidad hacia el actual gobierno y hacia las instituciones representativas del sistema político.

Consideramos que los problemas de legitimidad, y debilidad institucional, podrían comprometer el devenir de la Convención Constitucional. Contra las altas expectativas depositadas en esta iniciativa, el problema mencionado vuelve a acercar a Chile al vecindario donde exitosos procesos constituyentes como el colombiano, boliviano, ecuatoriano, entre otros, no han podido resolver satisfactoriamente el 
malestar y falta de legitimidad de las instituciones. Lo anterior plantea enormes desafíos a la administración vigente y a las futuras autoridades políticas a elegir el 21 de noviembre, así como también a la propia Convención Constitucional. Del comportamiento y el compromiso de los convencionales, y de otros actores relevantes, dependerá que el proceso sea o no la oportunidad para generar instancias y soluciones que permitan enfrentar, y en lo posible revertir, los problemas que han venido afectando a la institucionalidad democrática.

\section{REFERENCIAS}

Altman, D. y Luna, J. P. (2011). Chile: ¿institucionalización con pies de barro?. En M. A. Cameron y J. P. Luna (Eds.). Democracia en la región andina (pp. 273-313). IEP. https://doi. org/10.7440/2012.47

Arzola, M. P. (2019). Gratuidad y financiamiento de la educación superior: Cifras para el debate. Fundación Libertad y Desarrollo.

Avendaño, O. (2013). Reformas políticas en el gobierno de Sebastián Piñera, Chile 2010-2013. Revista Mexicana de Ciencias Políticas y Sociales. 58(218), 167-191. https://doi.org/10.1016/ S0185-1918(13)72295-5

AvendAÑo, O. (2014). Fracturas y representación política en el movimiento estudiantil. Chile 2011. Última Década (41), 41-68. http://doi. org/10.4067/S0718-22362014000200003

Avendaño, O. (2017). Entre diagnóstico crítico y propuestas de cambio. En M. A. Vera (Ed.). Malestar social y desigualdades en Chile (pp. 19-53). Ediciones Universidad Alberto Hurtado.

Avendaño, O. (2019). Estallido social en Chile: Los dilemas políticos desde octubre de 2019. Política. Revista de Ciencia Política. 57(2), 105-119. https:// doi.org/10.5354/0719-5338.2019.61558

Avendaño, O. y Escudero, M. C. (2019). La oposición a los gobiernos de centro-izquierda y de derecha. Una aproximación desde la experiencia chilena, 2006-2019. En L. de Oliveira X. y C. F. Domínguez A. (Eds.). Política, Cultura e Sociedade na América Latina: Estudos Interdisciplinares e Comparativos (pp. 271-295). Vol. 6. Editorial CRV.

Avendaño, O. y Escudero, M. C. (2020). Estallido social, crisis política en Chile y solución constitucional. Lecciones a partir de los acontecimientos del 18 de octubre de 2019. Ecuador Debate. (109), 139-156.

Barómetro del Trabajo (2020). Informe de participa- ción electoral plebiscito 26 abril. Fundación FIEL y CERC-MORI

BAsAdRe, G. (2018, 19 de diciembre). Muerte de Catrillanca: CIPER revela en exclusiva tres de los videos que grabó Carabineros. CIPER. https:// www.ciperchile.cl/2018/12/19/muerte-de-catrillanca-ciper-revela-en-exclusiva-tres-de-los-videos-que-grabo-carabineros/

Bellel, C. (2016). Dificultades y resistencias de una reforma para desmercantilizar la educación. Revista de la Asociación de Sociología de la Educación. 9(2), 232-247.

BRINKS, D., LeVITSKY, S. y MuriLlo, M. V. (2019). Understanding Institutional Weakness. Power and Design in Latin American Institutions. Cambridge University Press. https://doi.org/10.1017/9781108772211

COFRÉ, G. (2016). Aproximación a las movilizaciones regionalistas en el Chile actual: Los casos de Aysén, Calama y Magallanes [Tesis para optar al título profesional de Sociólogo, Universidad de Chile].

CRITERIA (2020). Agenda ciudadana Criteria febrero 2020. Criteria.

DATA INfluye (2020). Plebiscito, proceso constituyente y otras percepciones ciudadanas. Data Influye, enero.

Donoso, S. (2017). "Outsider" and "Insider" Strategies: Chile's Student Movement, 1990-2014. En S. Donoso y M. von Bülow (Eds.). Social Movements in Chile. Organization, Trajectories \& Political Consequences (pp. 65-97). Palgrave. https:// doi.org/10.1057/978-1-137-60013-4_3

Escudero, M. C. y Gajardo, J. (2020). Nueva Constitución y proceso constituyente. IdeAs. Idées d'Ameriques. (15), 1-7. https://doi.org/10.4000/ ideas.8417

Escudero, M. C., Márquez, R. e Irarrázaval, I. (2020). Etapa participativa del proceso constituyente chileno 2016. La experiencia del Comité de Sistematización. En R. Araya (Ed.). Doscientas mil voces. Ecos y aprendizajes del proceso constituyente del gobierno de Michelle Bachelet (pp. 195-210). Ediciones Abierta.

Escudero, M. C. (2021). Making a Constituent Assembly Possible in Chile: The Shifting Costs of Opposing Change. Bulletin for Latin American Research (en prensa) https://doi.org/10.1002/ BLAR.13290

Escudero, M. C. y Olivares, A. (2021). From the Dance of Those Left Out to New Constitution: Channeling the Chilean Social Unrest. En B. Navarrete y V. Tricot (Eds.). The Social Outburst and Political Representation in Chile. (pp. 173-192). 
Springer. https://doi.org/10.1007/978-3-03070320-2_10

FERnández, P. (2020). Sobre la marcha. Notas acerca del estallido social en Chile. Debate.

Fundación Sol (2016). Educar para mercantilizar. Fundación Sol

Gamboa, R., Escudero, M. C., Morales, M., Olivares, A. y Salcedo, R. (2016). La cultura política de los partidos. Una línea de base en la implementación de las reformas políticas. Ministerio Secretaría General de la Presidencia.

GǘLL, P. (2019). El estallido social de Chile: piezas para un rompecabezas. Revista Mensaje, (685), 10-15.

Huneeus, C. (2014). La democracia semisoberana. Chile después de Pinochet. Taurus.

Huneeus, C. y Avendaño, O. (2018). "Los partidos políticos y su debilitamiento". En C. Huneeus y 0. Avendaño. El sistema político de Chile (pp. 153191). Lom Ediciones.

LARRondo, P. (2019, 05 de octubre). Explican por qué aumentará la tarifa del transporte en la RM: "No es una buena noticia" para los pasajeros. EMOL. https://www.emol.com/noticias/Nacional/ 2019/10/05/963355/Metro-Transantiagotarifa-transporte-publico.html

Mahoney, J. y Thelen, K. (2010). A Theory of Gradual Institutional Change. En J. Mahoney y K. Thelen (Eds.). Explaining Institutional Change. Ambiguity, Agency, and Power (pp. 1-37). Cambridge University Press. https://doi.org/10.1017/ CB09780511806414.003

MARDones, R. (2007). Chile. "Todas íbamos a ser reinas". Revista de Ciencia Política. Vol. 27 (número especial), 79-96. http://doi.org/10.4067/ S0718-090X2007000100005.

Marol, A. (2012). No al lucro. De la crisis del modelo a la nueva era política. Debate.

Ministerio de Educación (2018, 20 de septiembre). Gobierno envía al Congreso Proyecto de Ley "Aula Segura". MINEDUC. https://www.mineduc.cl/ proyecto-de-ley-aula-segura/

Ministerio del Interior y Seguridad Pública (2019, 20 de octubre). Extiende declaración de Estado de Excepción Constitucional de Emergencia a toda la Región Metropolitana. Diario Oficial. https:// www.interior.gob.cl/transparenciaactiva/doc/ActosyDocumentosDiarioOficial/200/6849284.pdf

Miranda, N. y M. A. López (2020). Elecciones generales chilenas de 2017: el regreso de Piñera y el debut del nuevo sistema electoral. En M. Alcántara (Dir.). América Latina vota (2017-2019) (pp. 101-135). Tecnos.

Morales, M. (2018). Elecciones y participación en
Chile, 1988-2017. En C. Huneeus y O. Avendaño (Eds.). El sistema político de Chile (pp. 225257). Lom Ediciones.

Moreno, E. (2018). Años de transformaciones (Segundo gobierno de Michelle Bachelet). San José Impresores.

Moulian, T. (2006). Fracturas. De Pedro Aguirre Cerda a Salvador Allende (1938-1973). Lom Ediciones.

Moulian, T. (2020). El estallido social en Chile desde una perspectiva histórica. En A. Osorio (Ed.). América Latina en llamas: malestar, movilizaciones, estallidos sociales (pp. 16-22). RIIR.

OCDE (2018). Estudios económicos de la OCE, Chile. Better Policies for Better Lives.

Osorio Rauld, A. (2019a) iQue se vayan todos, que no quede uno solo! La crisis institucional en Chile: factores contributivos del socavamiento de las bases culturales de la democracia (1990-2019). Revista de investigaciones Políticas y Sociológicas. 18(2), 73-94. https://doi.org/10.15304/ rips.18.2.6375

Osorio Rauld, A. (2019b). Las actitudes políticas de la élite empresarial chilena hacia la democracia en un contexto de una crisis. Política. Revista de Ciencia Política. 57(2), 7-27. https://doi. org/10.5354/0719-5338.2019.61554

Osorio Rauld, A. (2019c). La cultura política de la élite empresarial chilena. Un análisis comparado de los dirigentes gremiales de la SNA y la SOFOFA (2018-2019) [Tesis doctoral, Universidad Complutense de Madrid]. https://eprints.ucm. es/id/eprint/59130/

Osses, B. (2019, 12 de noviembre). Presidente Piñera llama a acuerdos por la paz, la justicia y una nueva Constitución. EMOL. https://www.emol. com/noticias/Nacional/2019/11/12/967033/ Presidente-Pinera-acuerdos-manifestaciones-Constitucion.html

Peña, C. (2020). Pensar el malestar. La crisis de octubre y la cuestión constitucional. Taurus.

PNUD (1998). Desarrollo Humano. Las paradojas de la modernización. Programa de Naciones Unidas para el Desarrollo.

PNUD (2017a). Desiguales. Orígenes, cambios y desafíos de la brecha social en Chile. Programa de Naciones Unidas para el Desarrollo.

PNUD (2017b). Diagnóstico sobre la participación electoral en Chile. Programa de Naciones Unidas para el Desarrollo.

PNUD (2019). Diez años de auditoria a la democracia: Antes del estallido. Programa de Naciones Unidas para el Desarrollo.

RASSE, A. (2016). Segregación residencial socioeco- 
nómica y desigualdad en las ciudades chilenas. Documento de Trabajo $N^{\circ} 4$. Programa de $\mathrm{Na}$ ciones Unidas para el Desarrollo.

Romero, M. (2015). El poder ciudadano de Magallanes y la batalla del gas del 2011. En E. Valenzuela (Eds.). Territorios rebeldes. Autonomías versus presicracia centralista (pp. 407-434). Ediciones Universidad Alberto Hurtado.

Romo, S. (2020, 28 de septiembre). Frente Amplio ahora se abre a primarias con el resto de la oposición, pero exige "mínimos programáticos y éticos”. El Mostrador. https://www.elmostrador. cl/dia/2020/09/28/frente-amplio-ahora-seabre-a-primarias-con-el-resto-de-la-oposicionpero-exige-minimos-programaticos-y-eticos/

Ruiz, C. (2020). Octubre chileno. La irrupción de un nuevo pueblo. Taurus.

SalAzAR, G. (1990). Violencia política popular en las "grandes alamedas". Santiago de Chile 19471987. Ediciones SUR.

Segovia, C. y Gamboa, R. (2012). Chile: El año en que salimos a la calle. Revista de Ciencia Política, 32(1), 65-85. http://doi.org/10.4067/S0718090X2012000100004.

SiLva, E. (2016). Patagonia, without Dams! Lessons of a David vs. Goliath campaign. The Extractive Industries and Society. 3 (4), 947-957. https:// doi.org/10.1016/j.exis.2016.10.004

Sото, K. (2014, 05 de julio). UDI en marcha con apoderados de subvencionados: "Queremos reforma, pero no de esta forma”. La Tercera. https://www. latercera.com/noticia/udi-en-marcha-con-apoderados-de-subvencionados-queremos-reforma-pero-no-de-esta-forma/

TIRoni, E. (2020). El desborde. Vislumbres y aprendizajes del 18-0. Editorial Planeta.

URETA, S. (2017). Transantiago o el fallido ensamblaje de una sociedad de clase mundial. Ediciones Universidad Alberto Hurtado.

Varas, A. (2013). El gobierno de Piñera 2010-2014. La fronda aristocrática revivida. Catalonia.

Venegas, J. I. (2016). ¿Por qué los jóvenes chilenos rechazan la política? Desafección política juvenil en el Chile postransición. RIL Editores.

Vergara, L. (2019). Vivienda social y segregación: Una aproximación para entender las diferencias entre grandes y pequeñas ciudades. Revista AUS. (25), 26-31. https://doi.org/10.4206/ aus.2019.n25-05

Viera, C. (2020). El problema constitucional chileno. ¿Superar el Estado subsidiario? Revista de Ciencia Política. 58 (2), 49-63. https://doi. org/10.5354/0719-5338.2021.64150
Villarroel, M. J. (2019, 19 de octubre). 20 estaciones quemadas y 41 con diversos daños: el recuento de Metro por jornadas de protestas. Biobio Chile. https://www.biobiochile.cl/noticias/nacional/region-metropolitana/2019/10/19/20-estaciones-quemadas-y-41-con-diversos-danos-el-recuento-de-metro-por-jornadas-de-protestas.shtml 